\title{
Exploiting Spectrum Heterogeneity in Dynamic Spectrum Market
}

\author{
Alexander W. Min, Member, IEEE, Xinyu Zhang, Member, IEEE, \\ Jaehyuk Choi, Member, IEEE, and Kang G. Shin, Fellow, IEEE
}

\begin{abstract}
The dynamic spectrum market (DSM) is a key economic vehicle for realizing the opportunistic spectrum access that will mitigate the anticipated spectrum-scarcity problem. DSM allows legacy spectrum owners to lease their channels to unlicensed spectrum consumers (or secondary users) in order to increase their revenue and improve spectrum utilization. In DSM, determining the optimal spectrum leasing price is an important yet challenging problem that requires a comprehensive understanding of market participants' interests and interactions. In this paper, we study spectrum pricing competition in a duopoly DSM, where two wireless service providers (WSPs) lease spectrum access rights, and secondary users (SUs) purchase the spectrum use to maximize their utility. We identify two essential, but previously overlooked, properties of DSM: 1) heterogeneous spectrum resources at WSPs and 2) spectrum sharing among SUs. We demonstrate the impact of spectrum heterogeneity via an in-depth measurement study using a software-defined radio (SDR) testbed. We then study the impacts of spectrum heterogeneity on WSPs' optimal pricing and SUs' WSP selection strategies using a systematic three-step approach. First, we study how spectrum sharing among SUs subscribed to the same WSP affects the SUs' achievable utility. Then, we derive the SUs' optimal WSP selection strategy that maximizes their payoff, given the heterogeneous spectrum propagation characteristics and prices. We analyze how individual SU preferences affect market evolution and prove the market convergence to a mean-field limit, even though SUs make local decisions. Finally, given the market evolution, we formulate the WSPs' pricing strategies in a duopoly DSM as a noncooperative game and identify its Nash equilibrium points. We find that the equilibrium price and its uniqueness depend on the SUs' geographical density and spectrum propagation characteristics. Our analytical framework reveals the impact of spectrum heterogeneity in a real-world DSM, and can be used as a guideline for the WSPs' pricing strategies.
\end{abstract}

Index Terms-Cognitive radios, dynamic spectrum market, game theory, spectrum heterogeneity, spectrum pricing

\section{INTRODUCTION}

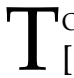
O mitigate the impending spectrum-scarcity problem [1], there have been continual efforts to deregulate wireless spectrum resources, and promote dynamic spectrum access (DSA). Recently, the FCC has opened up the TV spectrum band, allowing unlicensed devices to opportunistically access it as long as the unlicensed users do not interfere with legacy users' communications [2]. Meanwhile, various standardization efforts, such as the IEEE 802.22 WRANs [3], and IEEE 802.11af (a.k.a. Super Wi-Fi) [4] are being developed to utilize such spectrum white spaces. However, licensed users, referred to as primary users (PUs), have been reluctant to share their licensed spectrum because of 1) concerns of interference from

- A.W. Min is with the Circuits and Systems Research/System Architecture Lab, Intel Labs, 2111 N.E. 25th Avenue, Hillsboro, OR 97124. E-mail: alexander.w.min@intel.com.

- X. Zhang is with the Department of Electrical and Computer Engineering, University of Wisconsin-Madison, 1415 Engineering Dr., Madison, WI 53706. E-mail: xyzhang@ece.wisc.edu.

- K.G. Shin is with the Real Time Computing Laboratory (RTCL), Department of Electrical Engineering and Computer Science, The University of Michigan, 2260 Hayward Street, Ann Arbor, MI 481092121.E-mail:kgshin@eecs.umich.edu.

- J. Choi is with the Department of Design and Management, Gachon University, Soojeong-gu, Seongnam 461-701, Korea.

E-mail: jchoi@gachon.ac.kr.

Manuscript received 24 Feb. 2011; revised 22 Sept. 2011; accepted 30 Sept. 2011; published online 20 Oct. 2011.

For information on obtaining reprints of this article, please send e-mail to: tmc@computer.org, and reference IEEECS Log Number TMC-2011-02-0095. Digital Object Identifier no. 10.1109/TMC.2011.229. (unlicensed) secondary users (SUs) that can lead to potential loss of profit and 2) the lack of attractive incentives for PUs to share their licensed spectrum bands. The fear of interference can be overcome via recent advances in spectrum sensing technologies [5], [6], [7], [8] and proposals for a geolocation database [9], [10]. Thus, in order to realize the potential benefits of DSA, we need to construct effective mechanisms that incentivize the (licensed) spectrum owners to share spectrum resources with SUs.

The dynamic spectrum market (DSM) will play a key role in realizing DSA by facilitating spectrum trading between legacy spectrum owners and secondary consumers. ${ }^{1}$ This spectrum trading can be encouraged by a suitable pricing model through which DSM provides attractive economic incentives to legacy spectrum owners, and cost-effective spectrum access to secondary consumers. This will, in turn, enable more efficient and flexible usage of spectrum resources. Such a DSM already exists in various forms, such as mobile virtual network operators (MVNOs) [11] and online spectrum markets (e.g., specex . com [12]).

Interactions among DSM participants can be modeled as a 3-tier structure [13], [14] (see Fig. 1) consisting of: 1) the spectrum plane, where licensed spectrums are auctioned and sold to wireless service providers (WSPs), 2) the service plane, where WSPs sublease the spectrum by enticing SUs with competitive prices and good spectrum quality, and 3) the user

1. We use the terms spectrum consumer and secondary user interchangeably throughout the paper. 
plane, where SUs choose the WSP that maximizes their utility. Although spectrum pricing competition in DSM has been studied extensively [15], [16], [17], [18], most existing work has not considered spectrum heterogeneity as a primary factor in establishing the pricing strategy (except [19]).

A wide range of heterogeneous frequency bands will be available in a DSM considering the current trend of deregulating wireless resources. For example, the TV white space recently opened for unlicensed usage spans a wide range of frequencies over the VHF/UHF bands. Given this availability, it is natural for WSPs to want heterogeneous spectrum bands so as to avoid the interference between them. Due to the difference in propagation profile (i.e., frequency-dependent attenuation rate), heterogeneous channels have different transmission and interference ranges. For example, the lower the frequency band, the better the signal propagation characteristics at the same transmission power level. Rational secondary consumers would be able to evaluate the value/utility of different channels and exploit the capability of their software-defined radios (SDRs) to access the different ranges of spectrum bands available in the market.

Another important but largely overlooked feature of DSM is the necessity of sharing leased spectrum bands with other SUs, which is a common feature of wireless communications. This feature has implications in establishing the way market participants interact with each other. In a DSM, WSPs sublease their spectrum resources to multiple SUs in the same geographical area to maximize their revenue, exploiting the spatial reusability of wireless spectrum resources. Such spectrum sharing complicates the spectrum price-demand relationship, making the DSM different from the traditional market where goods are owned exclusively by buyers [20]. For example, when SUs share a leased channel, quoting a low spectrum price will lead to paradoxical results: A low price may attract more users, but it will also increase the level of interference among SUs, thus discouraging SUs from accessing it even at a low price. Therefore, understanding this price-demand relationship is of great importance to the design of WSPs' optimal spectrum pricing and SUs' WSP selection strategies.

In this paper, we propose a new spectrum-pricing model in a DSM, where, in order to maximize their profits, WSPs compete with heterogeneous spectrum resources-channels with disparate center frequencies and propagation profiles. In our model, we assume the availability of a wide range of heterogeneous bands in the spectrum plane, and analyze the spectrum pricing-demand relationship between WSPs (in the service plane) and SUs (in the user plane). In the user plane, SUs sublease and share the spectrum that provides the maximum utility. These features-spectrum heterogeneity and spectrum sharing-are essential for us to understand the WSPs' pricing competition in a DSM, but have not been explored well.

We formulate WSPs' pricing competition as a noncooperative game, taking into account the SUs' desire to maximize their utility. Here, "utility" refers to spectrum consumers' judgments about the tradeoff between achievable capacity and spectrum leasing cost. We examine the existence and uniqueness of the spectrum price Nash equilibrium
(NE), which depends upon SU density (i.e., total spectrum demand $^{2}$ ) and spectrum heterogeneity. Our investigation into the effects of three essential features-1) spectrum heterogeneity, 2) spectrum sharing among SUs, and 3) total spectrum demand (i.e., SU density)—provides useful insights and practical guidelines for designing spectrum pricing and purchase strategies in DSM.

In summary, this paper makes the following contributions:

- Introduction of a new DSM model where WSPs with heterogeneous spectrum resources compete for a higher market share. We demonstrate the impact of spectrum heterogeneity via in-depth measurements on a GNU Radio/USRP testbed. To the best of our knowledge, this is the first attempt to analyze the impact of spectrum heterogeneity in a DSM.

- Investigation of a new spectrum price-demand model based on the desire of SUs to maximize their utility, by evaluating the impact of spectrum heterogeneity, spatial spectrum sharing, and total spectrum demand.

- Derivation of SUs' optimal WSP selection strategies based on a mean-field approach to study how spectrum heterogeneity affects market equilibrium. Our mean-field approach simplifies the market model using a set of differential equations, and is shown to efficiently approximate an exact model using large-dimension Markov chains.

- Modeling of the pricing strategies among WSPs as a noncooperative game and identification of the key factors that influence the NE points, taking into account the price-demand relation caused by the utility maximizing behavior of SUs.

The remainder of this paper is organized as follows: Section 2 describes the duopoly DSM model and formulates the pricing game among WSPs as a noncooperative game. Section 3 shows the impact of spectrum heterogeneity via indepth measurements on a SDR testbed. Section 4 analyzes the impact of SU density on their achievable utility by analyzing mutual interference among SUs. Section 5 studies the SUs' optimal WSP selection strategies that maximize achievable utility. Section 6 derives the WSPs' optimal spectrum pricing strategies based on a realistic pricedemand function. Section 7 reviews existing work for spectrum pricing in a DSM. Finally, Section 8 concludes the paper and discusses future research issues.

\section{System Model and Assumptions}

In this section, we first present the DSM model and a signal propagation model which will be used throughout the paper. We then define the utility functions of SUs and WSPs. Finally, we formulate the pricing competition of WSPs as a non-cooperative game.

\subsection{A Dynamic Spectrum Market Model}

We consider a duopoly DSM where two WSPs compete in the same geographical area, as illustrated in Fig. 1. Each WSP

2. We refer to "spectrum demand" as the number of SUs in a DSM rather than the SUs' bandwidth demand. 


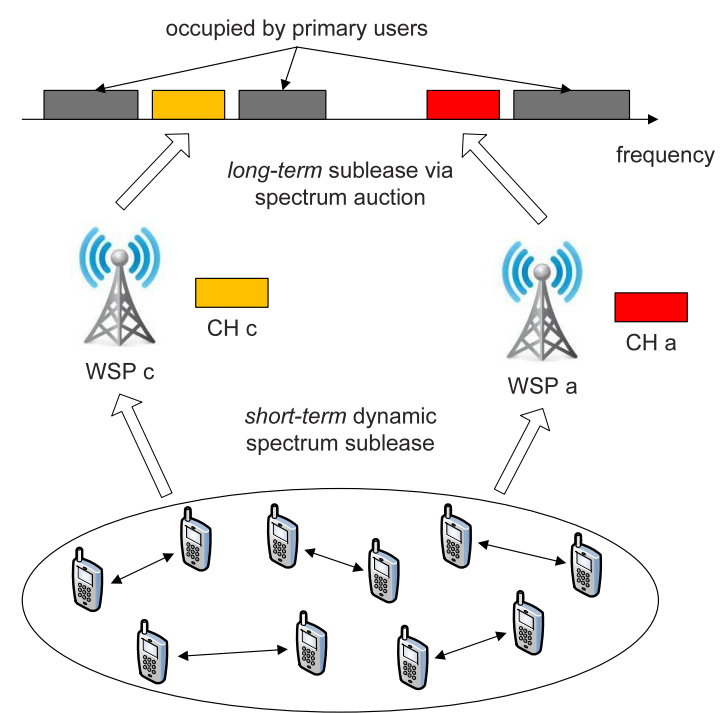

Fig. 1. A duopoly dynamic spectrum market model: WSPs compete with heterogeneous channels (leased from primary spectrum owners) to entice more SUs in the same geographical area in order to maximize profit.

is assumed to have long-term access rights for a licensed channel with a different center frequency, obtained from primary spectrum owners, for example, via auction [21], [22]. WSPs then grant access rights to their channels to multiple SUs by advertising the spectrum price, either via database query or direct broadcasting over a dedicated control channel. WSPs have access to complete information about the customer population (i.e., SU density) and their preferences (i.e., SUs' utilities). ${ }^{3}$ Each WSP possesses a single channel for leasing, and we focus on the case in which the WSPs' leased channels have considerably different center frequencies, thus exhibiting disparate wireless signal propagation characteristics.

For the user plane, we consider an ad hoc secondary network consisting of a set, $\mathcal{N}$, of transmitter-receiver pairs, referred to as SUs. Each pair constitutes a basic unit for spectrum leasing; in essence, SUs purchase short-term rights to access the channels from a WSP at a fixed spectrum price set by the WSP. We assume that SUs are SDR devices (e.g., USRP [23]) with cognitive radio capability. By exploiting the ability to access a wide range of spectrum bands, SUs aim to maximize their utility (i.e., the difference between channel capacity and spectrum leasing cost in (2)) by choosing the "best" WSP. SUs are randomly deployed in areas following a point Poisson process [24], [25] with average density $\rho$, i.e., the distribution of the number of active links within the deployment area, $A$, is $n_{A} \sim \operatorname{Poisson}(n ; \rho|A|)$. Note that although we consider an ad hoc secondary network, our analysis can also be applied to an infrastructure-based network model, where communication between an access point (or base station) and its associated clients is one-to-one at any given time.

\subsection{Signal Propagation and Spectrum Reuse Model}

Signal propagation is known to be affected by the center frequency of each channel: the lower the frequency band, the

3. Learning mechanisms can be used to infer such information when it is not available [19]. better the signal propagation characteristics. For ease of analysis without losing key insights to be gained from spectrum heterogeneity, we consider the following simple signal propagation model that reflects the impact of spectrum heterogeneity [26]:

$$
P_{R}=P_{o} g_{c}(r)=P_{o}\left(\frac{c_{o}}{f_{c}}\right)^{\alpha} r^{-\alpha}, \quad \text { (Watts) }
$$

where $P_{R}$ is the received signal power, $P_{o}$ the transmission power, $c_{o}$ the speed of light, i.e., $c_{o}=3 \times 10^{8} \mathrm{~m} / \mathrm{s}, f_{c}$ the center frequency of the channel $c, r$ the distance between the transmitter and receiver, and $\alpha(>2)$ the path-loss exponent. ${ }^{4}$ We assume that all the SUs in the network use the same fixed transmission power level $P_{o}$. While we use a simple signal propagation model, more realistic models (e.g., [27]) could be used for specific wireless environments (e.g., indoor or outdoor) at the cost of complexity of analysis. Since shadow or multipath fading is shown not to affect average interference significantly [28], we do not consider it in our model.

Buddhikot [29] suggested three different models for spectrum sharing, which are referred to as exclusive use, shared use, and commons models. These models overcome the limitations of the traditional command-and-control model. In order to focus on the impacts of spectrum heterogeneity in a DSM, in this paper we consider the exclusive use model, in which primary spectrum owners grant their exclusive spectrum access rights to a third party (e.g., WSPs). This exclusive model is suitable for spectrum bands with relatively long ON/OFF primary activity periods, e.g., DTV channels. Besides, this model can provide high quality-of-service (QoS) and reliability because it does not require frequent performance of spectrum sensing by SUs, or frequent service interruptions due to primary activities. Interested readers are referred to [29].

\subsection{Utility-Maximizing Spectrum Demand and User Preference}

One of our main contributions is to derive a realistic pricedemand function in the DSM, driven by SUs' desire to maximize their utility. Specifically, the utility function of SU $i \in \mathcal{N}$, which is associated with WSP (channel) $c^{5}$ is defined as the difference between the SUs' achievable link capacity and spectrum price:

$$
\mathcal{U}_{i}(c)=B \log \left(1+\frac{P_{o} g_{c, i}}{I_{c, i}+N_{o}}\right)-p_{c}
$$

where $B$ is the channel bandwidth, $g_{c, i}$ the channel gain between the secondary transmitter and receiver, $N_{o}$ the noise power level, and $P_{o}$ the transmit power. (Per FCC regulation, there is a cap on transmit-power levels for SUs.) We consider a fixed (unit) bandwidth demand from SUs, i.e., $B=1$ for all channels. The average of cumulative interference power caused by the SUs on channel $c$ at the receiver of link $i$ is denoted by $I_{c, i}$, and $p_{c}$ denotes the spectrum price (per unit time). To simplify the analysis, we assume that all the secondary transmitter-receiver pairs are separated by the same distance, and thus the channel gain $g_{c, i}$ only depends on channel frequency, i.e., $g_{c, i}=g_{c} \forall i$.

4. We assume that the path-loss exponent is $\alpha>2$ so that cumulative interference does not diverge as the network size grows.

5. We equate a WSP with its channel(s). 
For a similar reason, we assume $I_{c, i}=I_{c} \forall i$. Henceforth, we omit the subscript $i$ for brevity.

Let $\mathcal{C}=\{c, a\}$ denote the set of WSPs (channels) in a DSM. Based on the utility function in (2), SU $i$ selects the WSP (channel) $c_{i} \in \mathcal{C}$ that maximizes expected utility, i.e.,

$$
c_{i}^{*}=\arg \max _{c \in \mathcal{C}} \mathcal{U}_{i}(c) .
$$

\subsection{Spectrum Pricing Game between WSPs}

The main objective of WSPs is to maximize their profit by leasing the licensed channel to multiple SUs at the highest possible leasing price. Therefore, WSPs play a pricing game to compete for market share. The payoff (profit) function of a WSP $c \in \mathcal{C}$ is defined as: ${ }^{6}$

$$
\mathcal{V}_{c}\left(p_{c}, p_{-c}\right)=N_{c}\left(p_{c}, p_{-c}\right) \cdot p_{c}-b_{c},
$$

where $N_{c}$ is the number of SUs associated with WSP $c$ (spectrum demand), $p_{c}$ the spectrum leasing price, and $b_{c}$ the fixed investment cost, i.e., the fee paid to the primary spectrum owner for the long-term spectrum lease (per unit time).

Note that analyzing the price-demand relationship, i.e., $N_{c}\left(p_{c}, p_{-c}\right)$, is not straightforward. Traditional economic models tend to assume a known relation between WSPs' prices and SUs' demand. However, in our model, the spectrum demand $N_{c}$, i.e., the number of SUs on channel $c$, depends not only on WSPs' spectrum leasing prices $\left\{p_{c}, p_{-c}\right\}$, but also on the channel quality (capacity) determined by the frequency-dependent cochannel interference, as shown in (2). SUs can freely choose the WSP that maximizes their payoff. Thus, WSPs must consider spectrum heterogeneity in devising an optimal spectrum pricing strategy that maximizes profit.

Based on the WSPs' utilities in (4), the spectrum pricing game among WSPs can be defined as shown below.

Definition 1 (Spectrum pricing game between WSPs). $A$ spectrum pricing game between the WSPs can be formalized as a strategic choice:

$$
p_{c}^{*}=\arg \max _{p_{c} \in \mathbb{R}} \mathcal{V}_{c}\left(p_{c}, p_{-c}\right),
$$

where $p_{-c}$ denotes the price chosen by the competing WSP.

In what follows, we first demonstrate the impact of spectrum heterogeneity in Section 3, then analyze SU utility in Section 4, and derive the optimal WSP selection and spectrum pricing strategies in Sections 5 and 6, respectively.

\section{Characteristics of Spectrum Heterogeneity}

In this section, we demonstrate the effects of spectrum heterogeneity on received signal strength (RSS) via measurements on our SDR testbed. We first describe our experimental setup and then present the measurement results.

\subsection{Experimental Setup}

To evaluate the impact of spectrum heterogeneity, we constructed a GNU Radio/USRP2 [30] testbed on the fourth

6. Let the subscript $-c$ denote the competitor of WSP $c$.

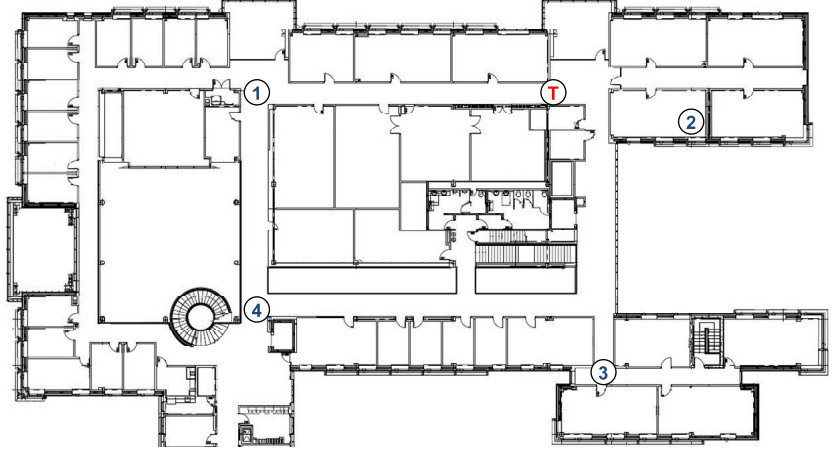

Fig. 2. Software-defined radio testbed: GNU Radio/USRP2 nodes were placed at different locations on the fourth floor of the CSE Building at the University of Michigan.

floor of the Computer Science and Engineering (CSE) Building at the University of Michigan. This floor has multiple offices and conference rooms and relatively straight corridors, which allow us to evaluate the impact of spectrum heterogeneity under both line-of-sight (LOS) and non-line-of-sight (NLOS) settings.

We deployed five USRP2 nodes in the topology shown in Fig. 2. We placed the transmitter at a fixed location in the corridor (denoted as circled $\mathrm{T}$ in the figure), and purposely placed four receiver nodes at different locations (e.g., corridors and offices, denoted as 1-4 in the figure) to test various signal-propagation environments. The measurements were done at night to minimize the effects of environmental changes, such as moving people/obstacles and interference from other networks. This allows us to focus on evaluation of the impact of spectrum heterogeneity on network performance without the need to deal with transient network dynamics, e.g., the fluctuations in RSS due to moving obstacles.

We equipped the USRP2 nodes with two different sets of daughterboards and antennae that operate on different spectrum bands. For high-frequency spectrum, we mounted the VERT2450 (dual band 2,400-2,480 MHz and 4.9-5.9 GHz omnidirectional antenna) on a XCVR2450 board (2.4-2.5 and 4.9-5.85 GHz dual-band daughterboard). For low-frequency bands, we mounted the VERT900 (824-960 MHz omnidirectional antenna) on a WBX board (50 MHz to $2.2 \mathrm{GHz}$ daughterboard). Both the XCVR2450 and WBX have the same transmit power level (20 dBm).

We used the benchmark DBPSK encoding/decoding module in GNU Radio to test the signal quality on different spectrum bands. The bit rate was set to $0.1 \mathrm{Mbps}$ and each BPSK symbol went through a raised-root-cosine filter with eight taps, resulting in a signal bandwidth of $50 \mathrm{KHz}$. Through experiments, we found that the transmit power of the testbed increases linearly with transmit gain. Therefore, we set the transmission gain of both XCVR2450 and WBX to the maximum, to ensure that they have the same output power.

To evaluate the effect of spectrum heterogeneity, we measured the signal-to-noise ratio (SNR) of a transmitted signal on three different frequency bands, i.e., $907 \mathrm{MHz}$, $2.478,5.728 \mathrm{GHz}$, at four different receiver locations. Receiver location 1 was LOS setting, and the rest were NLOS settings. The measurement lasted 5 minutes for each 


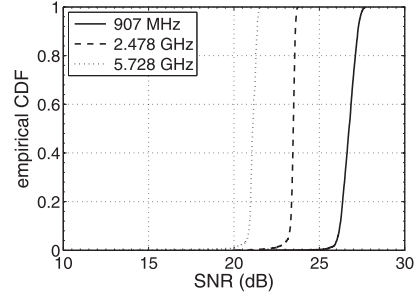

(a) Location 1 (LOS)

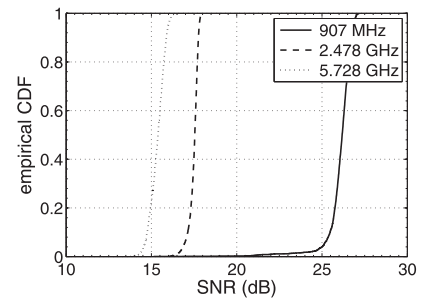

(c) Location 3 (NLOS)

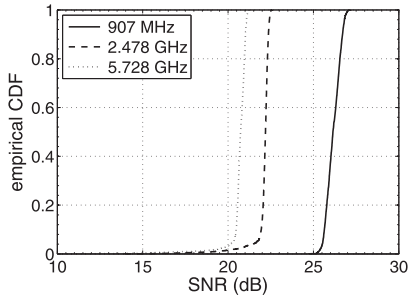

(b) Location 2 (NLOS)

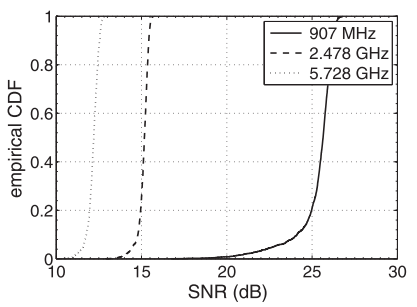

(d) Location 4 (NLOS)
Fig. 3. Impact of spectrum heterogeneity. The distribution of measured SNR depends significantly on the center frequency of the channel; the lower the frequency, the higher the SNR due to the better signal propagation characteristics.

experiment. Note that the USRP RF circuits have different gains for different frequency bands. Hence, we first calibrated the output power for different frequency bands, so that they would have comparable SNRs at short distances. In this way, the hardware artifacts were isolated and for each link, the signal quality only depended on its frequency.

\subsection{Experimental Results}

Fig. 3 plots the empirical cumulative distribution function (c.d.f.) of the measured SNR. The figure clearly indicates the impact of spectrum heterogeneity: the lower the frequency, the higher the SNR, regardless of the receiver locations. Fig. $3 \mathrm{~d}$ shows that, when the receiver is in the NLOS setting, high frequency bands, i.e., 2.478 and $5.728 \mathrm{GHz}$, suffer from significant deterioration in signal strength because of obstacles (i.e., the walls between the transmitter and receiver). On the other hand, the low-frequency band, i.e., $907 \mathrm{MHz}$, achieves a relatively high SNR thanks to its good wall-penetration characteristics.

Next, we studied the signal propagation characteristics of different spectrum bands by measuring the RSS (in $\mathrm{dB}$ ). We placed the transmitter at a fixed location and varied the transmitter-receiver separation from 15 to $45 \mathrm{~m}$ in an indoor, LOS setting. Fig. 4 illustrates that the low-frequency band shows consistent advantage for all the distance settings. In addition, RSS linearly decreases when the logarithmic distance, i.e., $10 \log _{10}(r)$, between the transmitter-receiver pair increases, regardless of the center frequency. This again verifies the trend predicted by the empirical propagation model in (1).

\section{ANALYSIS OF SECONDARY UtILITY UNDER SPECTRUM Heterogeneity}

In this section, we characterize cochannel interference (i.e., $I_{c, i}$ in (2)) among SUs to capture the effects of spectrum

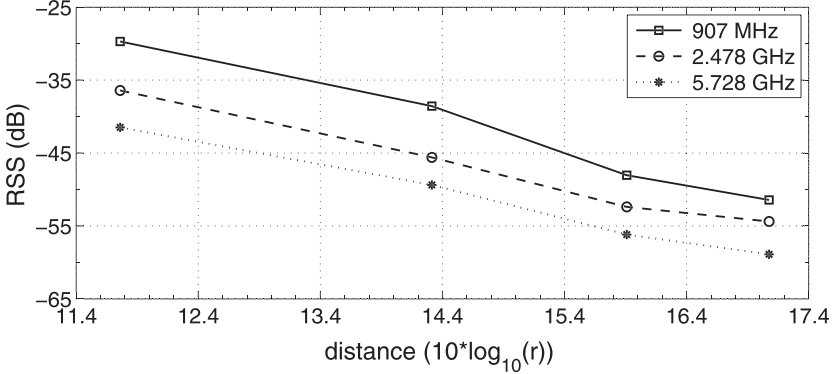

Fig. 4. Signal propagation over heterogeneous spectrum: RSS decreases almost linearly as the logarithmic distance between the transmitter and receiver increases.

heterogeneity and spectrum sharing on the achievable capacity of SUs.

For spectrum sharing among cochannel SUs, we consider the physical model in [31] where all the SUs can transmit at the same time, rather than the protocol model in [31]. ${ }^{7}$ Note that, although we consider the physical model, the main insights would not be different for the protocol model. We approximate the distribution of cochannel interference, $I_{c}$, on channel $c \in \mathcal{C}$, by quantifying the interference from SUs located inside and outside the interference range, $R_{I}^{c}$, which is defined as $R_{I}^{c} \triangleq \sup \left\{r \in \mathbb{R} \mid P_{o} g_{c}(r)>\eta\right\}$ where $\eta$ is a predefined threshold that depends on the desired data rate, modulation scheme, etc.

We first approximate the sum of cochannel interference caused by SUs located inside the interference range as a Gaussian random variable. In practice, secondary systems maintain a certain distance between them to avoid interference, so we assume that the minimum distance between secondary transmitters is sufficiently large (e.g., $>10 \mathrm{~m}$ ). The total interference at a fixed point in a uniformly distributed wireless network can be accurately approximated as a Gaussian random variable [32].

Let $\mathcal{G}_{i n, c}=\sum_{S_{c}} g_{c}(r)$ denote the normalized interference (i.e., sum of channel gains) from a set $S_{c}$ of cochannel SUs located inside the interference range. Then, the probability density function (p.d.f.) of Gaussian random variable $\mathcal{G}_{i n, c} \sim$ $N\left(\mu_{c}, \sigma_{c}^{2}\right)$ is given as:

$$
\mathcal{G}_{i n, c}(x)=\frac{1}{\sqrt{2 \pi}} \exp \left(-\frac{\left(x-\mu_{c}\right)^{2}}{2 \sigma_{c}^{2}}\right),
$$

where the mean $\left(m_{1}\right)$ and variance $\left(m_{2}\right)$ of the interference $\mathcal{G}_{i n, c}$ is given as [33]:

$$
\begin{aligned}
m_{k}(\rho, c) & =\rho_{c} \pi\left(\left(R_{I}^{c}\right)^{2}-\epsilon^{2}\right) \int_{\epsilon}^{R_{I}^{c}} \frac{2 r}{\left(\left(R_{I}^{c}\right)^{2}-\epsilon^{2}\right)}\left(g_{c}(r)\right)^{k} \mathrm{~d} r \\
& =\frac{2 \rho_{c} \pi}{(k \alpha-2)}\left(\frac{c_{o}}{f_{c}}\right)^{\alpha k}\left(\frac{1}{\epsilon^{k \alpha-2}}-\frac{1}{\left(R_{I}^{c}\right)^{k \alpha-2}}\right),
\end{aligned}
$$

where $\epsilon$ is the minimum separation distance from the receiver.

7. The physical and protocol models [31] are most widely-used for modeling wireless interference. In the former, SUs can transmit data concurrently but share the channel via a nonorthogonal multiplexing protocol (e.g., CDMA). In the latter, SUs multiplex the channels using an orthogonal scheme (e.g., OFDMA), and the per-user capacity is inversely proportional to the number of interfering neighbors. 
We now quantify the total interference caused by SUs located outside the interference range.

Lemma 1. The total interference caused by SUs on channel $c$ located outside the interference region (i.e., unit disk of radius $R_{I}^{c}$ centered at the receiver) can be approximated as:

$$
I_{\text {out }, c}=2 \pi P_{o}\left(\frac{c_{o}}{f_{c}}\right)^{\alpha} \frac{\rho_{c}\left(R_{I}^{c}\right)^{2-\alpha}}{\alpha-2} .
$$

Proof. See Appendix A, which can be found on the Computer Society Digital Library at http: / doi.ieeecomputersociety. org/10.1109/TMC.2011.229.

Finally, based on (7) and (8), the interference caused by SUs on channel $c$ can be approximated as $I_{c} \sim \mathcal{N}\left(\mu_{c}, \sigma_{c}^{2}\right)$ where

$$
\begin{gathered}
\mu_{c}=E\left[I_{\text {in, }, c}\right]+I_{\text {out }, c}=\left(\frac{c_{o}}{f_{c}}\right)^{\alpha} \frac{2 \pi \rho_{c} P_{o}}{\alpha-2}\left(\frac{1}{\epsilon^{\alpha-2}}\right), \\
\sigma_{c}^{2}=\frac{\rho_{c} \pi P_{o}}{\alpha-1}\left(\frac{c_{o}}{f_{c}}\right)^{2 \alpha}\left(\frac{1}{\epsilon^{2 \alpha-2}}-\frac{1}{\left(R_{I}^{c}\right)^{2 \alpha-2}}\right),
\end{gathered}
$$

where $\rho_{c}$ is the density of SUs (links) on channel $c$. Similarly, we can derive interference for channel $a$ (i.e., $\mu_{a}$ and $\sigma_{a}^{2}$ ). Equation (9) indicates that the total interference linearly increases with SU density $\rho_{c}$, which can be approximated as $\rho_{c} \approx \frac{N_{c}}{|A|}$ where $A$ is the entire network area.

The interference distribution in (9) and (10) is a function of center frequency $f_{c}$, which serves as the basis for developing an optimal WSP selection strategy for analyzing the pricing game among WSPs with heterogeneous spectrum bands.

\section{Optimal WSP Selection Strategy via MEAN-FIELD APPROACH}

In this section, we derive the optimal WSP selection strategy for SUs using a mean-field approach, assuming that the WSPs possess different spectrum bands. We begin with a mean-field approximation for the evolution of the spectrum market. We then prove its convergence, and derive the optimal WSP selection strategy in the mean-field regime.

\subsection{A Mean-Field Model for the Spectrum Market}

The mean-field method [34] is a simple and effective way of analyzing the state evolution of a large number of interacting objects. In particular, it is suitable for analyzing how the local behavior of individual nodes affects the global properties of a large-scale network. In our problem, an SU's behavior is described by its type (i.e., its preferred WSP), and the global properties are the steady-state distribution of SU types.

Our mean-field approach uses differential equations to approximate the evolution of the market, whose state converges to the fixed point of the equation (namely, the mean-field limit) under certain conditions [34]. In what follows, we first use a mean-field model to describe how the DSM evolves, and then justify the convergence of the market to its mean-field.

\subsection{Evolution and Convergence of the Market}

We first provide the following key definitions:

- A link is defined as a connected transmitter-receiver pair with active traffic. Therefore, a link can be considered "newly joined" if it has just switched from an idle period to a period of bursty transmission.

- Let $N$ be the number of active links. Links can "join" and "depart" according to a Poisson distribution. However, we assume that the link population evolves to a steady state, such that the departure rate equals the arrival rate, and the total number of links remains roughly constant.

- Let $\lambda$ be the traffic rate of a link. We also assume that the ON-OFF traffic pattern of a link is bursty, following a Poisson distribution with rate $\lambda$.

- Let $N_{c}(t)$ denote the total number of active links using channel $c$ at time $t$. Links are classified according to the channel that they use, i.e., a link $i$ is of type $c$, if it selects channel $c \in \mathcal{C}$.

We study the evolution of the spectrum market within a short period of time, $\Delta t$. The number of newly joined secondary links within this period is $N \lambda \Delta t$. This is also the number of departed links within $\Delta t$, since we focus on a steady state of the SU population when the departure rate equals the arrival rate. Each newly joined link leases a channel from a WSP with a short-term contract. Note that active links that have already leased a channel are in transmitting/receiving mode, and must maintain their current channel (WSP) selection.

Let $P_{c}$ be the probability that, for a randomly selected link $i$, channel $c$ provides the maximum utility, i.e.,

$$
P_{c}=\operatorname{Pr}\left\{c=\arg \max _{c^{*} \in \mathcal{C}} \mathcal{U}_{i}\left(c^{*}\right)\right\}, \quad \forall c \in \mathcal{C},
$$

where the utility $\mathcal{U}_{i}(c)$ is defined in (2). Then, among the newly joined links within $\Delta t$, the number of links selecting channel $c$ is $N \lambda \Delta t P_{c}$.

The total number of channel $c$ SUs, i.e., links using channel $c$, in the network at time $(t+\Delta t)$ is

$$
N_{c}(t+\Delta t)=N_{c}(t)+N \lambda \Delta t P_{c}-N_{c}(t) \lambda \Delta t .
$$

Equation (12) describes the evolution of a market. The market equilibrium can be defined as a fixed point of the market evolution:

$$
\begin{gathered}
\frac{\partial N_{c}(t)}{\partial t}=\frac{N_{c}(t+\Delta t)-N_{c}(t)}{\Delta t}=N \lambda P_{c}-N_{c}(t) \lambda=0 \\
\Longleftrightarrow P_{c}=\frac{N_{c}(t)}{N} .
\end{gathered}
$$

Equation (13) indicates that the probability that an SU selects WSP $c$ is equivalent to the fraction of SUs using channel $c$, which is referred to as the channel occupancy measure, i.e., $\Pi_{c}(t)=N_{c}(t) / N$. Intuitively, the occupancy measure, $\Pi_{c}(t)$, reflects the market share of WSP $c$ at time $t$.

Proposition 1 (Convergence of channel occupancy). The channel-occupancy measure $\Pi=\left\{\Pi_{a}, \Pi_{c}\right\}$ converges to a deterministic process in the continuous-time domain.

Proof. See Appendix B, available in the online supplemental material. 
From now on, we will focus on deriving the channel (WSP) selection probability $P_{c}$ in the mean-field model of (12), which depends primarily on three key factors: 1) amount of interference $I_{c}$ on channel $c, 2$ ) spectrum leasing prices $p_{c}$, and 3) total spectrum demand $\rho$. Note that the interference intensity $I_{c}$ depends on the occupancy measure of channel $c$, which, in turn, affects the channel-selection probability $P_{c}$. This circular dependency eventually converges to a fixed point, i.e., the mean-field limit of market dynamics.

\subsection{SUs' Optimal Selection of WSPs}

We now analyze the SUs' optimal channel (WSP) selection strategies, assuming that each $\mathrm{SU}$ is a rational market entity which selects a WSP to maximize his utility. To make a strategic choice, each SU takes into account the achievable capacity and leasing cost, but cannot directly affect the price set by the WSPs. This model mirrors a real-world market economy where customers are obedient price-takers, but the joint effect of their choices causes the sellers to compete and reach an equilibrium price.

We derive the optimal WSP selection strategy in a meanfield regime for given spectrum prices $\mathbf{p}=\left\{p_{a}, p_{c}\right\}$. For an arbitrarily-chosen SU in a DSM, the probability that channel $c$ provides better utility is

$$
\begin{aligned}
P_{c} & =\operatorname{Pr}\left(\mathcal{U}_{c}-\mathcal{U}_{a}>0\right) \\
& =\operatorname{Pr}\left(\log \left(\frac{P_{o} g_{c}}{I_{c}+N_{o}}\right)-\log \left(\frac{P_{o} g_{a}}{I_{a}+N_{o}}\right)>p_{c}-p_{a}\right) \\
& =\operatorname{Pr}\left(\log \left(\frac{I_{a}+N_{o}}{I_{c}+N_{o}}\right)>p_{c}-p_{a}-\alpha \log \left(\frac{f_{a}}{f_{c}}\right)\right) \\
& =\operatorname{Pr}\left(I_{a}+N_{o}-e^{p_{c}-p_{a}}\left(\frac{f_{c}}{f_{a}}\right)^{\alpha}\left(I_{c}+N_{o}\right)>0\right),
\end{aligned}
$$

where $p_{c}\left(p_{a}\right)$ and $f_{c}\left(f_{a}\right)$ are the price and center frequency of channel $c(a)$, respectively.

Remark. Note that a more commonly used approach for analyzing the equilibrium state is to equate the user's utility, i.e., $\mathcal{U}_{i}(c)=\mathcal{U}_{i}(a)$ (e.g., [17]). However, such an equilibrium state may not be reached depending on the network environment, as will be shown in Section 6.4. Moreover, our approach can be easily extended to an oligopoly market, in which more than two WSPs compete with each other to entice SUs (see Section 5.5).

For given prices, the channel-selection probability $P_{c}$ depends solely on the interference statistics on channel $c$. In (14), the interference power on each channel can be approximated as a normal random variable as derived in (9) and (10) in Section 4.

Let $I_{c a}=I_{a}+N_{o}-\gamma_{c a}\left(I_{c}+N_{o}\right)$ where $\gamma_{c a}=e^{p_{c}-p_{a}}\left(\frac{f_{c}}{f_{a}}\right)^{\alpha}$. Note that $N_{o}$ and $\gamma_{c a}$ are constants, and $I_{c a}$ is thus the difference between the two Gaussian random variables, which is also Gaussian. Then, $I_{c a} \sim \mathcal{N}\left(\mu_{c a}, \sigma_{c a}^{2}\right)$ where

$$
\begin{gathered}
\mu_{c a}=\mu_{a}+N_{o}-\gamma_{c a}\left(\mu_{c}+N_{o}\right), \\
\sigma_{c a}^{2}=\sigma_{a}^{2}+\gamma_{c a}^{2} \sigma_{c}^{2},
\end{gathered}
$$

where the mean $\left(\mu_{c}\right)$ and variance $\left(\sigma_{c}^{2}\right)$ of the interference are shown in (9) and (10). Then, the channel-selection probability is

$$
P_{c}=\operatorname{Pr}\left(I_{c a}>0\right)=\operatorname{Pr}\left(\frac{I_{c a}-\mu_{c a}}{\sigma_{c a}}>\frac{-\mu_{c a}}{\sigma_{c a}}\right)=Q\left(\frac{-\mu_{c a}}{\sigma_{c a}}\right),
$$

where $Q(x)=\frac{1}{2 \pi} \int_{x}^{\infty} e^{-\frac{t^{2}}{2}} \mathrm{~d} t$. Using (9), (10), (16), and (17), one can derive the channel-selection probabilities.

Proposition 2 (WSP selection strategy). For the case with two WSPS (channels) $c$ and $a$, the mean-field limit of the channelselection strategy $P_{c}$ and $P_{a}$ follows (18) and (19).

$$
\begin{aligned}
P_{c} & =Q\left(\left\{\left(\frac{c_{o}}{f_{a}}\right)^{\alpha} \frac{2 \pi \rho P_{o}}{\alpha-2}\left(\frac{1}{\epsilon^{\alpha-2}}\right)\left(e^{p_{c}-p_{a}} P_{c}-P_{a}\right)\right.\right. \\
& \left.+N_{o}\left(e^{p_{c}-p_{a}}\left(\frac{f_{c}}{f_{a}}\right)^{\alpha}-1\right)\right\} /\left\{\left(\frac{c_{o}}{f_{a}}\right)^{\alpha} \sqrt{\frac{\pi \rho P_{o}}{\alpha-1}}\right. \\
& \sqrt{\left.\left.P_{a}\left(\frac{1}{\epsilon^{2 \alpha-2}}-\frac{1}{\left(R_{I}^{a}\right)^{2 \alpha-2}}\right)+e^{2\left(p_{c}-p_{a}\right)} P_{c}\left(\frac{1}{\epsilon^{2 \alpha-2}}-\frac{1}{\left(R_{I}^{c}\right)^{2 \alpha-2}}\right)\right\}\right),},
\end{aligned}
$$

$$
P_{a}=1-P_{c},
$$

Proposition 2 indicates that the mean-field limit of the WSP selection strategy is influenced not only by spectrum prices, but also by the channel heterogeneity reflected by interference ranges $\left(R_{I}^{c}, R_{I}^{a}\right)$ and center frequencies $\left(f_{c}, f_{a}\right)$. This clearly indicates that spectrum heterogeneity can affect the optimal spectrum pricing that maximizes the WSP's profit. Proposition 2, however, shows that SUs' traffic intensity $\lambda$ does not affect the system's steady-state.

\section{Proposition 3 (Asymptotic behavior of WSP selection} strategy). The optimal WSP selection probability becomes more uniform as SU density increases, i.e.,

$$
P_{c} \rightarrow 0.5 \quad \text { as } \quad \rho \rightarrow \infty,
$$

where $\rho$ is the average SU density, which can be approximated as $\rho \approx \frac{N}{|A|}$.

Proof. As $\rho \rightarrow \infty$, the WSP selection probability $P_{c}$ in (18) reduces to

$$
\lim _{\rho \rightarrow \infty} P_{c}=Q\left(+\infty\left(e^{p_{c}-p_{a}} P_{c}-P_{a}\right)\right) .
$$

Then, we have

$$
\lim _{\rho \rightarrow \infty} P_{c}= \begin{cases}1 & P_{c}<\frac{P_{a}}{e^{p_{a}-p_{a}}} \\ 0.5 & P_{c}=\frac{P_{a}}{e^{p_{a}-p_{a}}} \\ 0 & P_{c}>\frac{P_{a}}{e^{p_{a}-p_{a}}} .\end{cases}
$$

In (22), there exists a unique solution, i.e., $\lim _{\rho \rightarrow \infty} P_{c}=$ 0.5 when $p_{c}=p_{a}$. On the other hand, when $p_{c} \neq p_{a}$, there is no solution because $p_{c}=p_{a}$ is the unique NE point under the condition $\rho \rightarrow \infty$. We will detail the price NE in Section 6.

Proposition 3 indicates that the WSP selection probability becomes independent of spectrum heterogeneity when the number of SUs in the network, $N$, approaches infinity. This is because, when there exist a large number 


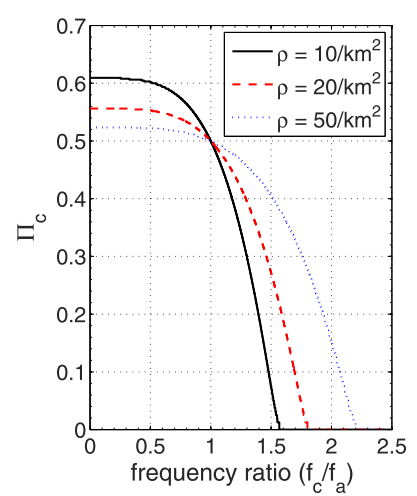

(a) Impact of spectrum heterogeneity

Fig. 5. Characterization of channel-occupancy measure: (a) The occupancy of channel $c, \Pi_{c}$, increases as the frequency ratio $\frac{f_{c}}{f_{a}}$ decreases, and (b) channel occupancy becomes less sensitive to spectrum heterogeneity as network density increases. The parameters are set to $\epsilon=100 \mathrm{~m}$, and $P_{o}=100 \mathrm{~mW}$, and spectrum prices are fixed at $p_{a}=p_{c}=1$.

of interferers, interference power dominates noise power, i.e., $I_{c} \gg N_{o}$, and as a result, the benefit from low frequency becomes negligible.

\subsection{Numerical Results}

Here, we present numerical results that show the behavior of the channel-occupancy measure under different DSM settings.

Fig. 5a shows the impact of heterogeneous channel frequencies on the channel occupancies, $\Pi_{a}$ and $\Pi_{c}$. In the simulations, we fix the center frequency of channel $a$ at $f_{a}=500 \mathrm{MHz}$ and increase the frequency $f_{c}$ up to $2.5 \mathrm{GHz}$. We set spectrum prices to $p_{a}=p_{c}=1$, to eliminate the effect of prices on channel occupancy. The figure shows that, when $f_{c}<f_{a}, \Pi_{c}>0.5$, due to the favorable signalpropagation characteristics of channel $c$; on the other hand, when $f_{c}>f_{a}, \Pi_{c}<0.5$ for the same reason. Interestingly, channel occupancy depends on average secondary network density (i.e., total spectrum demand) $\rho$. This is because, in a dense network where interference power exceeds noise power, i.e., $N_{o} \ll I_{c}$, the benefit of favorable signalpropagation characteristics diminishes. As a result, the channel-occupancy curve becomes flatter, confirming Proposition 3. Note that when $f_{c}=f_{a}, \Pi_{c}=\Pi_{a}=0.5$, regardless of SU density.

Fig. $5 b$ shows the channel-occupancy measure while varying average SU density in the range $\rho \in[0,200] / \mathrm{km}^{2}$. Here we fix the center frequencies at $f_{a}=500 \mathrm{MHz}$ and assume $f_{c} \in\{500 \mathrm{MHz}, 750 \mathrm{MHz}, 1 \mathrm{GHz}\}$. The figure indicates that the channel occupancy $\Pi_{a}$ is always greater than or equal to 0.5 due to its favorable signal-propagation characteristics. When SU density is low, the channel occupancy $\Pi_{a}$ is close to 1 as most SUs tend to enjoy the favorable signal-propagation characteristics of channel $a$ without worrying about mutual interference. Under these conditions, the DSM behaves monopolistically. However, as SU density increases, the channel-occupancy measure $\Pi_{a}$ decreases because, in such a high interference regime, it becomes harder for SUs to exploit the benefits of favorable signal-propagation characteristics. Thus, the DSM behaves like a duopoly. The figure also shows that the occupancy

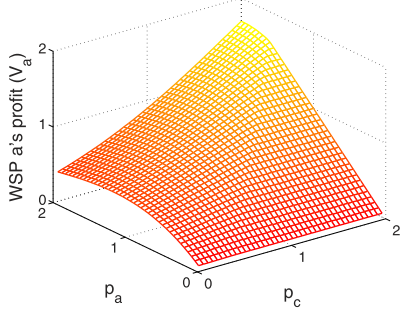

(a) Profit of WSP a $\left(\mathcal{V}_{a}\right)$

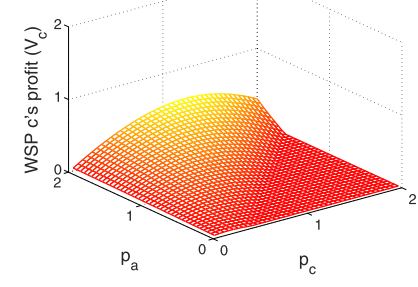

(b) Profit of WSP c $\left(\mathcal{V}_{c}\right)$
Fig. 6. Profit of WSPs: The achievable profit of WSPs depends on spectrum leasing prices $\mathbf{p}=\left(p_{a}, p_{c}\right)$ and spectrum heterogeneity (i.e., channel frequency). We fix the center frequencies at $f_{a}=500 \mathrm{MHz}$ and $f_{c}=1 \mathrm{GHz}$, and set SU density to $\rho=50 / \mathrm{km}^{2}$.

measure approaches 0.5 in all the tested cases, again confirming the correctness of Proposition 3.

\subsection{Optimal WSP Selection in Oligopoly DSM}

Although we primarily focus on a duopoly DSM, in realworld environments, there could be more than two WSPs that compete with each other, forming an oligopoly DSM. Our derivation of the optimal WSP-selection strategy in (14) can be easily extended to the oligopoly setting. Let us define the probability that WSP $j$ provides a higher utility than that of WSP $k$ as $P_{j k}=\operatorname{Pr}\left(\mathcal{U}_{j}>\mathcal{U}_{k}\right)$, and $P_{k j}=1-P_{j k}$.

Suppose there is a set $\mathcal{C}$ of WSPs where $|\mathcal{C}|>2$. Then, the probability that channel $j$ provides the highest expected utility can be calculated as:

$$
P_{j}=\frac{\prod_{k \in \mathcal{C} \backslash\{j\}} P_{j k}}{\sum_{s \in \mathcal{C}}\left(\prod_{t \in \mathcal{C} \backslash\{s\}} P_{s t}\right)}, \quad \forall j \in \mathcal{C},
$$

where $P_{j k}$ can be calculated using (18) and (19).

As we observed in the previous sections, the channelselection probability depends on multiple factors, such as spectrum heterogeneity, spectrum price, spectrum demand, etc., and no closed-form solution exists due to their complex interactions. However, (23) can serve as a basis to understand the spectrum pricing game in an oligopoly DSM, which is part of our future work.

\section{Equilibrium of the Spectrum-Pricing Game}

In this section, we study the impact of spectrum price on the WSP's profit as defined in (4), and characterize the NE points of pricing strategies.

\subsection{Impact of Spectrum Price on WSP's Profit}

Here we evaluate the impact of the WSPs' spectrum-pricing strategy $\mathbf{p}=\left(p_{a}, p_{c}\right)$ on their achievable profits. Without loss of generality, we set the heterogeneous spectrum bands at $f_{a}=500 \mathrm{MHz}$ and $f_{c}=1 \mathrm{GHz}$, i.e., the frequency of WSP $a$ is always lower than that of WSP $c$. However, we observed similar patterns for different frequency bands. We fixed SU density at $\rho=50 / \mathrm{km}^{2}$, and set the investment costs in (4) at $b_{a}=b_{c}=0$ to eliminate their impact on WSPs' profits, which will be studied separately in Section 6.6.

Fig. 6 shows that WSP $a$ always achieves a higher profit than WSP $c$, i.e., $\mathcal{V}_{a}>\mathcal{V}_{c}$, thanks to its favorable spectrum profile. Fig. 6a shows that the profit of WSP a (i.e., $\mathcal{V}_{a}$ ) monotonically increases as competing WSP $c$ increases its 


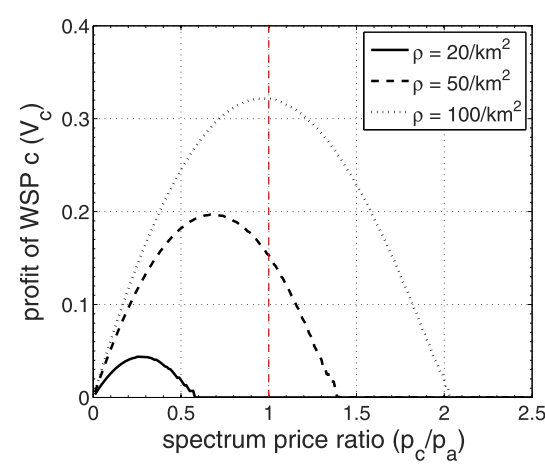

Fig. 7. Impact of price ratio: There exists an optimal pricing ratio that maximizes profit, and the effect of pricing is coupled with the SUs' density.

price $p_{c}$. This is because WSP $a$ tends to entice more customers due to channel $a^{\prime}$ s better signal-propagation characteristics. The advantage becomes more pronounced when the competitor WSP $c$ sets a higher price and loses part of its market share. In contrast, as shown in Fig. 6b, when WSP $c$ quotes a higher price than that of WSP $a$, its achievable profit remains 0 , i.e., WSP $a$ monopolizes the market. This indicates that channel $c$ is not competitive unless the price of channel $a$ rises above a certain threshold.

Fig. 7 shows the impact of relative price, $\frac{p_{c}}{p_{a}}$, on WSPs' profits with respect to $\mathrm{SU}$ density $\rho$ (i.e., spectrum demand). We set price $p_{a}=1$ and vary the price $p_{c}$ from 0 to 2.5. Here, we have made three observations. First, the relationship between price ratio and profit is a concave function as shown in Fig. 7. When the price ratio is relatively low, WSP $c^{\prime}$ 's profit decreases as the price ratio further decreases. Despite the fact that a lower price attracts more SUs, the advantage is limited by increased interference among them. When the ratio is relatively high, the profit also decreases as the ratio further increases, due to the significant decrease in the number of customers. Second, when the price ratio, $\frac{p_{c}}{p_{a}}$, is above a certain price threshold, the profit $\mathcal{V}_{c}$ becomes 0 (i.e., the profit curve becomes flat) since the high price makes channel $c$ unattractive to customers. However, such a threshold increases with an increasing SU density (i.e., spectrum demand) because WSPs can take advantage of a large number of customers. Third, in a sparse network with low SU density, profit $\mathcal{V}_{c}$ is maximized when $p_{c} \ll p_{a}$ because the interference on channel $a$ remains negligible, even when most users are associated with WSP $a$. In contrast, in a dense network, $\mathcal{V}_{c}$ is maximized when $p_{c} \approx p_{a}$ because all SUs will suffer from high interference regardless of the channel characteristics. Therefore, WSP $a$ loses its competitive advantage of superior signal propagation characteristics. Note that this corresponds to our findings in Proposition 3 in Section 5.3.

\subsection{Nash Equilibrium for Pricing Game}

In a DSM, WSPs must carefully set the spectrum price, since too high a price results in loss of market share, while too low a price will limit their achievable profits. We capture this tradeoff with the notion of NE.

Definition 2 (Spectrum price Nash equilibrium). $A N E$ in the duopoly game is defined as a strategy set $\left\{p_{c}^{*}, p_{a}^{*}\right\}$ that satisfies

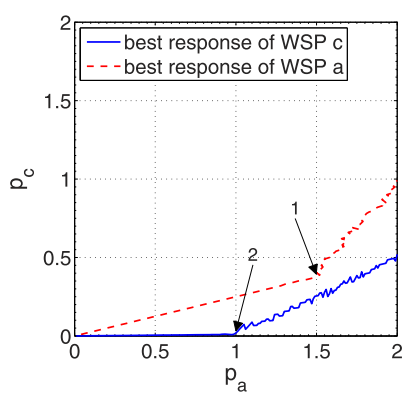

(a) $\rho=10 / \mathrm{km}^{2}$

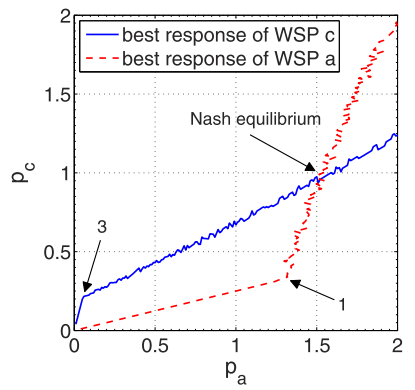

(c) $\rho=50 / \mathrm{km}^{2}$

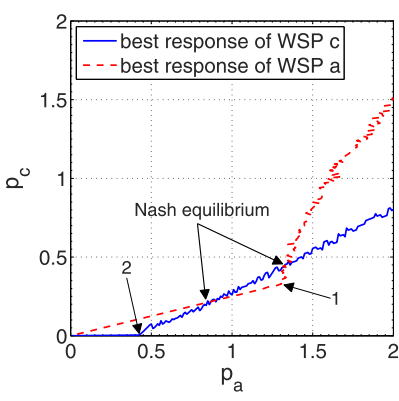

(b) $\rho=20 / \mathrm{km}^{2}$

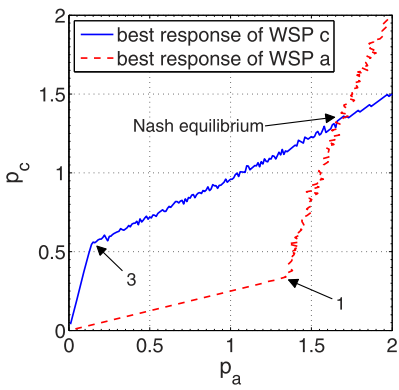

(d) $\rho=100 / \mathrm{km}^{2}$
Fig. 8. Best response functions for the WSPS: The existence and uniqueness of the NE depends on the spectrum heterogeneity as well as the secondary network density. In the simulation, we set $f_{a}=500 \mathrm{MHz}$, $f_{c}=1 \mathrm{GHz}$, and $b_{a}=b_{c}=0$.

$$
\begin{aligned}
& p_{c}^{*}=\arg \max _{p_{c}} \mathcal{V}_{c}\left(p_{c}, p_{a}^{*}\right), \\
& p_{a}^{*}=\arg \max _{p_{a}} \mathcal{V}_{a}\left(p_{c}^{*}, p_{a}\right) .
\end{aligned}
$$

Intuitively, an NE strategy set implies that no player can increase its profit by unilaterally adjusting the price. With the above definition, we can derive the NE of the duopoly game. Unfortunately, it is difficult to find a closed-form expression for the NE. Hence, we numerically solve (23) and (24) using a simple iterative search algorithm to obtain the NE price.

\subsection{Existence and Uniqueness of Nash Equilibrium}

Based on the above definition of $\mathrm{NE}$, we examine the existence and uniqueness of the NE points when SU density changes, which is equivalent to changing the spectrum demand over the entire network. In the simulation, we consider a representative scenario in which the frequency of WSP $a$ is lower than that of WSP $c$, i.e., $f_{a}=500 \mathrm{MHz}$ and $f_{c}=1 \mathrm{GHz}$, and thus, we expect the NE points to be formed such that $p_{a}^{*}>p_{c}^{*}$.

Fig. 8 shows the best responses for WSPs under different SU densities. We have made three key observations. First, the WSP $c^{\prime}$ s best response (solid lines) increases as the spectrum price $p_{a}$ increases, and vice versa. This is because WSPs compete over the same pool of customers in a given network coverage area, and hence, WSPs' optimal spectrum pricing is always relative to the competitors' spectrum prices. That is, if WSP $a$ quotes a high spectrum price, then the SUs' achievable utilities from WSP $a$ will decrease,

8. Although we presented the NEs for a specific set of frequencies, we observed from simulations a similar behavior for other frequency bands. 


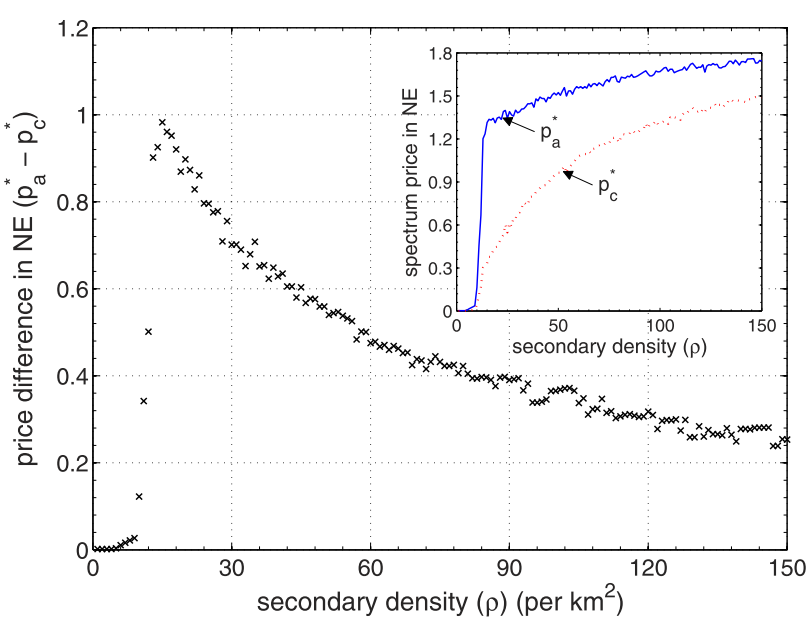

(a) Difference in NE prices

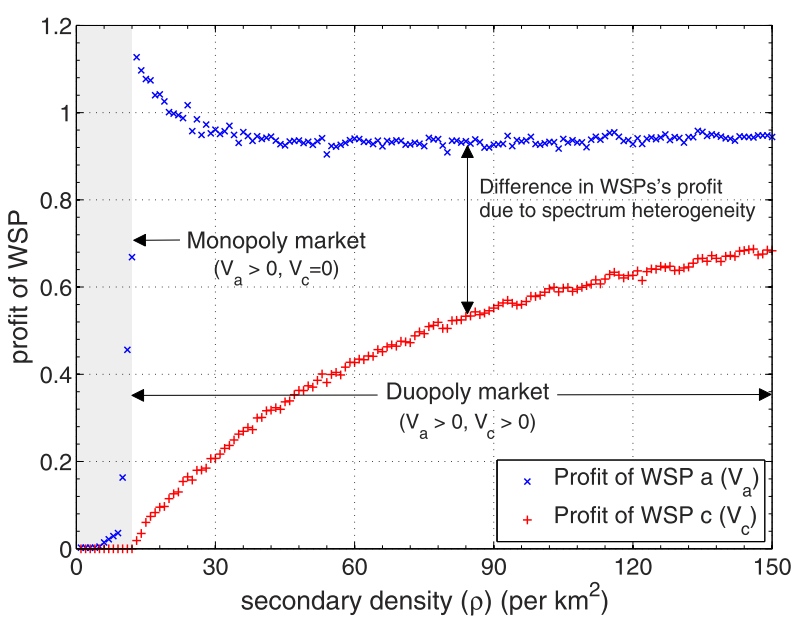

(b) Profit of WSPs

Fig. 9. Impact of secondary network density under heterogeneous and homogeneous spectrum bands: (a) The difference in NE prices, i.e., $p_{a}^{*}-p_{c}^{*}$, decreases with increasing SU density where $f_{a}=500 \mathrm{MHz}$ and $f_{c}=1 \mathrm{GHz}$, and (b) the NE prices increase with SU density. We assume zero investment cost, i.e., $b_{a}=b_{c}=0$, in the simulation.

changing their preference to the competitor, i.e., WSP $c$. This will allow WSP $c$ to increase its price $p_{c}$ to reach an equilibrium point.

Note that this relative behavior of spectrum pricing provides an economic incentive to WSPs for collusion. However, such collusion can be prevented in practice for the following reasons. There will be alternative technologies to access the wireless spectrum, e.g., IEEE 802.11, and hence, WSPs will lose their competitiveness as they advertise unreasonably high prices. Moreover, rational SUs would not purchase the spectrum if their achievable utility (i.e., difference between capacity and price) is too low, e.g., less than 0 . Therefore, WSPs cannot set spectrum prices arbitrarily to increase their profit.

Second, when SU density is low, i.e., $\rho=10 / \mathrm{km}^{2}$, the price NE does not exist because total spectrum demand is not high enough for WSPs to make a profit. Although $p_{c}=$ $p_{a}=0$ can also be considered as an NE point, the WSPs will avoid this strategy since this NE point will provide a negative revenue to both WSPs. That is, to attract customers, WSPs have to lower their prices until they reach 0 , and thus, there is no economic incentive for WSPs to participate in the market. In contrast, with high SU density, i.e., $\rho>20 / \mathrm{km}^{2}$, the NEs are formed at positive values, thus providing economic incentives to WSPs.

Third, Fig. 8 indicates that the best responses exhibit phase transitions (the transition thresholds denoted as 1, 2, $3)$, resulting in a different number of NEs depending on market settings. For example, the figures show that the growing rate of the best responses of WSP $a$ (dashed lines) changes at certain thresholds (denoted as 1). This is because when $p_{a}$ remains below the threshold, it is optimal for WSP $a$ to increase the price $p_{a}$ at a higher pace than $p_{c}$, i.e., $\frac{\Delta p_{a}}{\Delta p_{c}}>1$, to take advantage of channel $a^{\prime}$ s superior spectrum characteristics. However, when $p_{a}$ increases beyond the threshold, the high spectrum price limits the growth of the utility of SUs. As a result, channel $c$ becomes more attractive than channel $a$, and thus, $\frac{\Delta p_{a}}{\Delta p_{c}}<1$. Similarly, the best response of WSP $c$ has the threshold property denoted as 2 and 3 in the figures.
One interesting observation is that, in dense networks, i.e., $\rho=50,100 / \mathrm{km}^{2}$, the price $p_{c}$ increases faster than $p_{a}$ until $p_{a}$ reaches the threshold 3 . This is because, despite channel $a^{\prime}$ s higher quality, when the price $p_{a}$ is too low compared to the NE price, WSP $c$ can quote a higher price, i.e., $p_{c}>p_{a}$, to maximize its own profit, benefiting from a large number of customers.

\subsection{Market Dynamics under Various SU Densities}

As we observed in Section 5, SU density (or spectrum demand) is a critical factor in WSPs' pricing competition. Here, we investigate the impact of SU density on market dynamics by examining the NE prices, WSPs' profits, and SUs' utilities.

Fig. 9a shows the difference between the NE prices, i.e., $p_{a}^{*}-p_{c}^{*}$, as a function of $\mathrm{SU}$ density. When the density is low, i.e., $\rho<10 / \mathrm{km}^{2}$, NE does not exist as we observed in Fig. $8 \mathrm{a}$, and WSPs cannot make a profit because the market (spectrum demand) is too small. As the density increases, however, the NE price of channel $a\left(p_{a}^{*}\right)$ grows drastically, whereas the price $p_{c}^{*}$ remains 0 due to its inferior spectrum profile. This means WSP $c$ cannot make profit if they quote a price greater than $p_{c}>0$. As a result, WSP a monopolizes the market, as more clearly shown in Fig. 9b (shaded region). As the density increases further, however, WSP $c$ starts to share the market, i.e., duopoly, because the SUs on channel $a$ begin to suffer from cochannel interference.

Fig. 9b shows WSPs' profits defined in (4) for various SU densities. As we discussed, when density is low, WSP a dominates (monopolizes) the market, i.e., $\mathcal{V}_{a}>0$ and $\mathcal{V}_{c}=0$, thanks to its superior spectrum profile. As the SU density increases beyond a certain density threshold (i.e., $\rho=12 / \mathrm{km}^{2}$ ), the market becomes a duopoly and the difference in achievable profit decreases as the size of the market grows. Such a threshold density depends on spectrum heterogeneity. Fig. 10 clearly shows that the range of SU density below which WSP $a$ monopolizes the market increases as the center frequency of channel $c$ increases. For example, when $f_{c}=2 f_{a}=1 \mathrm{GHz}$, WSP $a$ will dominate the market until SU density becomes larger than $\rho=13 / \mathrm{km}^{2}$. In 


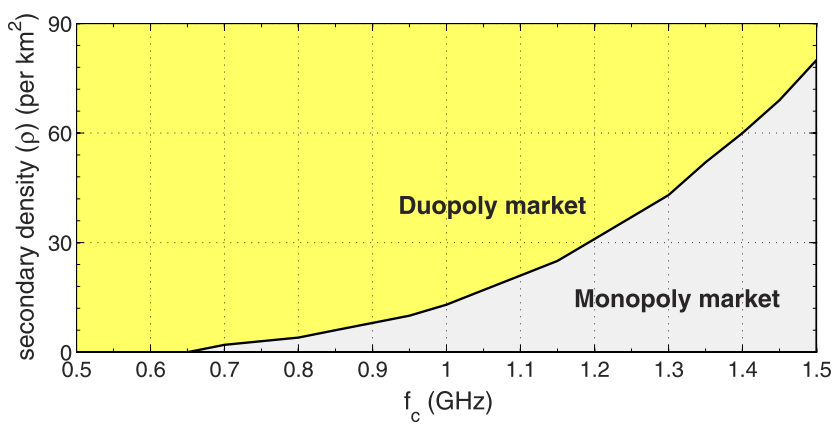

Fig. 10. Monopoly versus duopoly DSM: Market can be monopolized (gray area) by WSP $a$ when the channel frequency $f_{c}$ of the competitor WSP $c$, is relatively higher than $f_{a}$ and the SU density is low. We assume that $f_{a}$ is fixed at $500 \mathrm{MHz}$.

addition, such a boundary of SU density increases superlinearly, partly because of the relationship between received signal strength and center frequency, i.e., $P_{R} \propto f_{c}^{-\alpha}$, as indicated in (1).

Fig. 11 shows SUs' achievable utilities on each channel, i.e., $\mathcal{U}_{a}$ and $\mathcal{U}_{c}$. The figure shows that, when $\rho<13 / \mathrm{km}^{2}$, the utility on channel $a$ exceeds that of channel $c$, i.e., $\mathcal{U}_{a}>\mathcal{U}_{c}$, thus forming the monopoly market. On the other hand, in the duopoly market, there is no difference in achievable utilities, and thus the market is stabilized.

\subsection{Price NE under Spectrum Homogeneity}

To demonstrate the impact of SU density, while separating it from spectrum heterogeneity, we consider three homogeneous spectrum bands, i.e., $f_{a}, f_{c} \in\{500,600,700 \mathrm{MHz}\}$, and plot the corresponding NE points in Fig. 12. Due to spectrum homogeneity, the NE prices are equal, i.e., $p_{a}^{*}=p_{c}^{*}$, regardless of the SU density. We set the leasing cost $b_{a}=b_{c}=0$ to eliminate its impact on NE prices. From Fig. 12, we have two main observations. First, the equilibrium price increases with increasing SU density (i.e., total spectrum demand) due to the increasing number of customers. In addition, the lower the frequency band, the higher the price for any given SU density, since low-frequency bands return higher utility (i.e., capacity minus spectrum price) to the SUs. Second, the

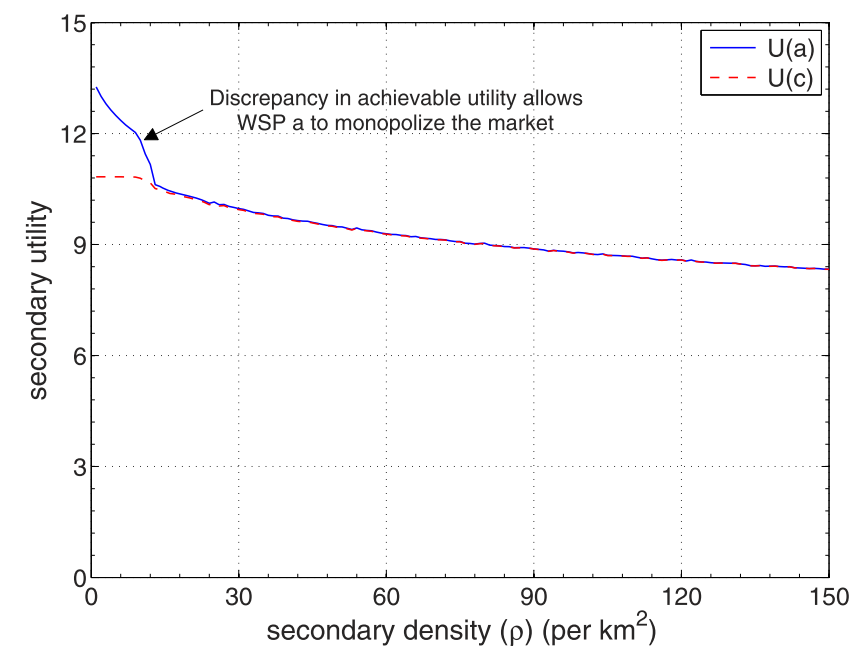

Fig. 11. Behavior of secondary utility: The achievable secondary utility differs only when SU density is low (i.e., monopoly market), and remains the same in the duopoly market.

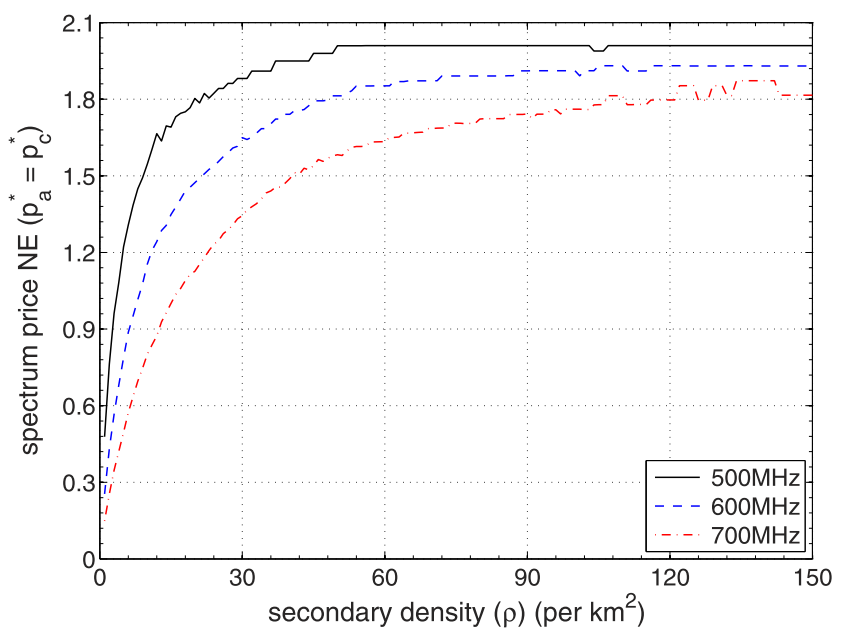

Fig. 12. The behavior of NE prices in a DSM with homogeneous spectrum bands: The equilibrium prices increase with SU density and converge at different rates; the lower the center frequency, the faster the convergence due to their large interference range.

equilibrium price converges faster with low-frequency bands due mainly to the large interference power (range) of low-frequency bands. This is because the potential benefit of using low-frequency bands (i.e., a longer transmission range) diminishes faster with SU density due to the large interference range.

\subsection{Impact of Spectrum Investment Cost}

Our analysis on WSPs' pricing game can provide a practical guideline for WSPs' spectrum investment decisions, such as a strategy for purchasing from the spectrum owners (e.g., via auction) in the spectrum plane, as shown in Fig. 1. Let us consider a spectrum market where WSP $a$ operates with a channel at frequency $f_{a}=500 \mathrm{MHz}$, which is obtained at cost $b_{a}=1$. Then, WSP $c$ ponders whether to join the market by purchasing a channel with $f_{c}$ from legacy spectrum owners at price $b_{c}$, which we refer to as spectrum investment cost.

Fig. 13 shows the maximum investment $\operatorname{cost} b_{c}^{\max }$, beyond which the profit becomes negative, i.e., WSP $c$ cannot make a profit in the market. The maximum investment cost

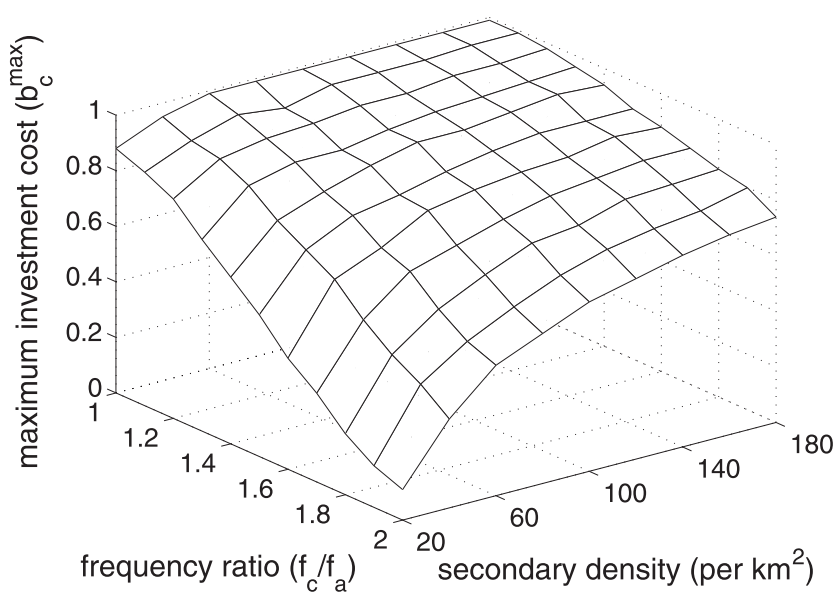

Fig. 13. Maximum investment cost. WSP $c$ 's maximum investment cost $b_{c}$ for making profit in the market is determined by the channel frequency $f_{c}$ and SU density. We assume $f_{a}=500 \mathrm{MHz}$ and $b_{a}=1$ for the competitor (i.e., WSP a). 
depends on spectrum heterogeneity as well as SU density. The figure indicates that the maximum investment cost $b_{c}^{\max }$ is always lower than $b_{a}=1$ due to channel $c$ 's inferior spectrum profile, but it approaches $b_{a}$ as the SU density increases in the market or channel $c$ has a better spectrum profile, i.e., a lower value of $f_{c}$.

\section{Related Work}

The problem of optimal spectrum pricing in the spectrum market has been studied extensively, and we discuss some of the work closely related to ours. Niyato and Hossain [16] analyzed spectrum pricing competition in cognitive radio networks with multiple primary service providers. Inaltekin et al. [15] considered heterogeneous channel conditions due to nodes' physical distances from the base station in wireless IP networks. Jia and Zhang [14] studied the duopoly wireless spectrum market where two WSPs compete for bandwidth and price to maximize their profit. Duan et al. [18] studied WSPs' investment and pricing mechanisms by considering SUs' physical-layer wireless characteristics. In [35], they also studied WSPs' optimal spectrum investment and pricing decisions in cognitive radio networks where spectrum availability dynamically changes due to the unpredictability of PUs' channel usage patterns. Gajić et al. [36] studied pricing competition among WSPs via a two-stage multi-leader-follower game. Mutlu et al. [37] studied measurement-based online pricing for secondary spectrum access and developed a pricing framework for an unknown demand function and call-length durations. However, none of the above studies considered the heterogeneity of a wide range of available spectrum bands in the spectrum market and spectrum sharing among colocated SUs in accessing the leased spectrum resources.

The closest to our study is [22] which considered two CRspecific features: 1) bandwidth (supply) uncertainty due to PUs' activities, and 2) spatial reuse of wireless spectrum. They studied an interesting market scenario where multiple WSPs compete with each other by jointly optimizing the spectrum price based on time and location-dependent spectrum availability. Such fine-grained coordination, however, might not be suitable for a highly dynamic wireless environment due to its high computation and communication overhead. In contrast, we assume a decentralized DSM where individual spectrum consumers purchase the payoffmaximizing spectrum, just as in a real-world market economy. Spectrum price stabilizes when multiple WSPs competing for market share reach a Nash equilibrium.

\section{Conclusion And Future Work}

The dynamic spectrum market is a promising paradigm to provide economic incentives that facilitate dynamic spectrum access. In this paper, we identified two key factors in a DSM-spectrum heterogeneity and spectrum sharing among SUs-and studied their impact on price competition among WSPs in a three-step approach. We first observed that SUs must share the wireless spectrum in a spatial domain, and established the effect of SU density (spectrum demand) on achievable utility when they are associated with the same WSP. We then derived the SUs' optimal WSP selection strategy that maximizes utility, for a given spectrum profile and leasing prices. Finally, we formulated
WSPs' spectrum pricing as a noncooperative game and identified its Nash equilibrium points. Our analysis demonstrates that spectrum heterogeneity significantly influences WSPs' spectrum pricing, especially in a sparse network. In a dense network, the benefit of a lower frequency band diminishes due to severe cochannel interference, and thus, spectrum heterogeneity has less impact on spectrum pricing.

In the future, we would like to investigate the impact of spectrum heterogeneity on WSPs' auction strategies in the spectrum plane. It would also be interesting to extend the analytical framework to a DSM with multiple WSPs. Moreover, we plan to study the dependency of an optimal spectrum price on other system parameters, e.g., maximum transmission power.

\section{ACKNOWLEDGMENTS}

The work reported in this paper was supported in part by the US National Science Foundation under Grants CNS1114837 and CNS-0721529 and by the US Defense Advanced Research Projects Agency (DARPA) under Grant HR0011-10-1-0081.

\section{REFERENCES}

[1] "Mobile Broadband Capacity Constraints and the Need for Optimization," http://www.rasavy.com/Articles/2010_02 Rysavy_Mobile_Broadband_Capacity_Constraints.pdf, 2012.

[2] FCC, "Second Memorandum Opinion and Order," FCC 10-174, Sept. 2010

[3] IEEE 802.22 Working Group on Wireless Regional Area Networks, http://www.ieee802.org/22, 2012.

[4] IEEE 802.22 Working Group on Wireless Local Area Networks, http://www.ieee802.org/11, 2012.

[5] M. Gandetto and C. Regazzoni, "Spectrum Sensing: A Distributed Approach for Cognitive Terminals," IEEE J. Selected Areas in Comm., vol. 25, no. 3, pp. 546-557, Apr. 2007.

[6] Q. Zhao, L. Tong, A. Swami, and Y. Chen, "Decentralized Cognitive MAC for Opportunistic Spectrum Access in Ad Hoc Networks: A POMDP Framework," IEEE J. Selected Areas in Comm., vol. 25, no. 3, pp. 589-600, Apr. 2007.

[7] A.W. Min and K.G. Shin, "An Optimal Sensing Framework Based on Spatial RSS-Profile in Cognitive Radio Networks," Proc. IEEE CS Sixth Ann. Sensor, Mesh, and Ad Hoc Comm. and Networks (SECON), June 2009.

[8] A.W. Min, K.G. Shin, and X. Hu, "Secure Cooperative Sensing in IEEE 802.22 WRANs Using Shadow Fading Correlation," IEEE Trans. Mobile Computing, vol. 10, no. 10, pp. 1434-1447, Oct. 2011.

[9] D. Gurney, G. Buchwald, L. Ecklund, S. Kuffner, and J. Grosspietsch, "Geo-Location Database Techniques for Incumbent Protection in the TV White Space," Proc. IEEE Third Symp. New Frontiers in Dynamic Spectrum Access Networks (DySPAN), Oct. 2008.

[10] R. Murty, R. Chandra, T. Moscibroda, and P. Bahl, "SenseLess: A Database-Driven White Spaces Network," Technical Report MSRTR-2010-127, Sept. 2010.

[11] http://en.wikipedia.org/wiki/Mobile_virtual_network_ operator, 2012

[12] http://www.specex.com, 2012.

[13] J.M. Chapin and W.H. Lehr, "The Path to Market Success for Dynamic Spectrum Access Technology," IEEE Comm. Mag., vol. 45, no. 5, pp. 96-103, May 2007.

[14] J. Jia and Q. Zhang, "Competitions and Dynamics of Duopoly Wireless Service Providers in Dynamic Spectrum Market," Proc. ACM MobiHoc, May 2008.

[15] H. Inaltekin, T. Wexler, and S.B. Wicker, "A Duopoly Pricing Game for Wireless IP Services," Proc. IEEE CS Fourth Ann. Sensor, Mesh, and Ad Hoc Comm. and Networks (SECON), June 2007.

[16] D. Niyato and E. Hossain, "Competitive Pricing for Spectrum Sharing in Cognitive Radio Networks: Dynamic Game, Inefficiency of Nash Equilibrium, and Collusion," IEEE J. Selected Areas in Comm., vol. 26, no. 1, pp. 192-202, Jan. 2008. 
[17] D. Niyato, E. Hossain, and Z. Han, "Dynamics of Multiple-Seller and Multiple-Buyer Spectrum Trading in Cognitive Radio Networks: A Game-Theoretic Modeling Approach," IEEE Trans. Mobile Computing, vol. 8, no. 8, pp. 1009-1022, Aug. 2009.

[18] L. Duan, J. Huang, and B. Shou, "Competition with Dynamic Spectrum Leasing," Proc. IEEE Symp. New Frontiers in Dynamic Spectrum Access Networks (DySPAN), Apr. 2010.

[19] Y. Xing, R. Chandramouli, and C. Cordeiro, "Price Dynamics in Competitive Agile Spectrum Access Markets," IEEE J. Selected Areas in Comm., vol. 25, no. 3, pp. 613-621, Apr. 2007.

[20] A. Mas-Colell, M. Whinston, and J. Green, Microeconomic Theory. Oxford Univ., 1995.

[21] X. Zhou, S. Gandhi, S. Suri, and H. Zheng, "eBay in the Sky: Strategy-Proof Wireless Spectrum Auctions," Proc. ACM MobiCom, Sept. 2008.

[22] G.S. Kasbekar and S. Sarkar, "Spectrum Pricing Games with Bandwidth Uncertainty and Spatial Reuse in Cognitive Radio Networks," Proc. ACM MobiHoc, Sept. 2010.

[23] USRP: Universal Software Radio Peripheral, http://www.ettus. com, 2012.

[24] C. Bettstetter, "On the Minimum Node Degree and Connectivity of a Wireless Multihop Network," Proc. ACM MobiHoc, June 2002.

[25] Y. Choi and S. Choi, "Service Charge and Energy-Aware Vertical Handoff in Integrated IEEE 802.16e/802.11 Networks," Proc. IEEE INFOCOM, May 2007.

[26] A. Goldsmith, Wireless Communications. Cambridge Univ., 2005.

[27] ITU-R Recommendation P.1546-3, Method for Point-to-Area Predictions for Terrestrial Services in the Frequency Range $30 \mathrm{MHz}$ to 3000 $\mathrm{MHz}, 2007$.

[28] X. Hong, C.-X. Wang, and J. Thompson, "Interference Modeling of Cognitive Radio Networks," Proc. IEEE Vehicular Technology Conf. (VTC-Spring), May 2008.

[29] M.M. Buddhikot, "Understanding Dynamic Spectrum Access: Models, Taxonomy and Challenges," Proc. IEEE Second Int'l Symp. New Frontiers in Dynamic Spectrum Access Networks (DySPAN), Apr. 2007.

[30] GNU Software Radio Project, http://www.gnu.org/software/ gnuradio, 2012.

[31] P. Gupta and P.R. Kumar, "The Capacity of Wireless Networks," IEEE Trans. Information Theory, vol. 46, no. 2, pp. 388-404, Mar. 2000.

[32] K.S. Gilhousen, I.M. Jacobs, R. Padovani, A.J. Viterbi, L.A. Weaver Jr, and C.E. Wheatley III, "On the Capacity of a Cellular CDMA System," IEEE Trans. Vehicular Technology, vol. 40, no. 2, pp. 303312, May 1991.

[33] R. Menon, R.M. Buehrer, and J.H. Reed, "On the Impact of Dynamic Spectrum Sharing Techniques on Legacy Radio Systems," IEEE Trans. Wireless Comm., vol. 7, no. 11, pp. 4198-4207, Nov. 2008.

[34] M. Benaim and J.-Y. L. Boudec, "A Class of Mean Field Interaction Models for Computer and Communication Systems," Performance Evaluation, vol. 65, nos. 11/12, pp. 823-838, Apr. 2008.

[35] L. Duan, J. Huang, and B. Shou, "Cognitive Mobile Virtual Network Operator: Investment and Pricing with Supply Uncertainty," Proc. IEEE INFOCOM, Mar. 2010.

[36] V. Gajić, J. Huang, and B. Rimoldi, "Competition of Wireless Providers for Atomic Users: Equilibrium and Social Optimality," Proc. 47th Ann. Allerton Conf. Comm., Control, and Computing, 2009.

[37] H. Mutlu, M. Alanyali, and D. Starobinski, "On-Line Pricing of Secondary Spectrum Access with Unknown Demand Function and Call Length Distribution," Proc. IEEE INFOCOM, Mar. 2008.

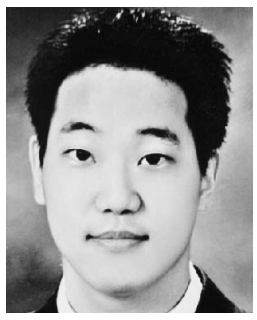

Alexander W. Min received the BS degree in electrical engineering from Seoul National University, Korea, in 2005 and the $\mathrm{PhD}$ degree in electrical engineering and computer science from the University of Michigan, Ann Arbor, in 2011. He is currently a research scientist in the System Architecture Lab at Intel Labs. In 2010, he was a research intern at Deutsche Telekom, Inc., R\&D Labs, Los Altos. His research interests are in the areas of cognitive radio and dynamic spectrum access networks, wireless security, low-power mobile platform, and mobile sensing. He has served as a reviewer for numerous journals and conferences in the wireless area and on technical program committees for IEEE PIMRC and IEEE ICC. He is a member of the IEEE, IEEE Communications Society, and the ACM.

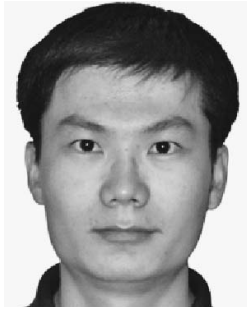

Xinyu Zhang received the BE degree in 2005 from the Harbin Institute of Technology, China, the MS degree in 2007 from the University of Toronto, Canada, and the PhD degree in 2012 from the University of Michigan-Ann Arbor. He is currently an assistant professor in the Department of Electrical and Computer Engineering at the University of Wisconsin-Madison. His research interest lies in designing protocols and algorithms that improve the capacity, interoperability, and energy efficiency of wireless networks. His work spans the areas of wireless networking, communications engineering, and mobile computing, involving both mathematical analysis and experimental validation on software-radio platforms. He was a recipient of the Best Paper Award at ACM MobiCom 2011. He is a member of the IEEE.

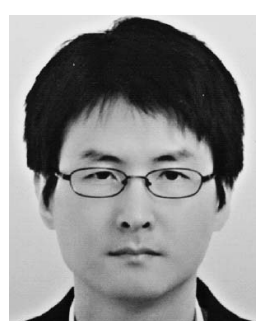

Jaehyuk Choi received the $\mathrm{PhD}$ degree in electrical engineering and computer science from Seoul National University, Korea, in 2008. $\mathrm{He}$ is currently an assistant professor in the Department of Software Design \& Management at Gachon University, Seongnam, Korea. From 2009 to 2011, he was a postdoctoral research fellow in the Department of Electrical Engineering and Computer Science at the University of Michigan, Ann Arbor. He was a postdoctoral fellow in Brain Korea 21 at Seoul National University in 2008. His current research interests are in the areas of wireless/mobile networks with emphasis on wireless LAN/MAN/PAN, network management, nextgeneration mobile networks, cognitive radios, data link layer protocols, and cross-layer approaches. He is a member of the IEEE.

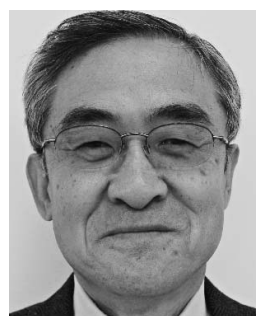

Kang G. Shin is the Kevin \& Nancy O'Connor professor of computer science in the Department of Electrical Engineering and Computer Science, The University of Michigan, Ann Arbor. His current research focuses on computing systems and networks as well as on embedded real-time and cyber-physical systems, all with emphasis on timeliness, security, and dependability. He has supervised the completion of $69 \mathrm{PhDs}$ and authored/coauthored more than 770 technical articles (more than 270 of these are in archival journals), one textbook, and more than 20 patents or invention disclosures, and received numerous awards, including Best Paper Awards from the 2011 ACM International Conference on Mobile Computing and Networking (MobiCom '11), the 2011 IEEE International Conference on Autonomic Computing, the 2010 and 2000 USENIX Annual Technical Conferences, as well as the 2003 IEEE Communications Society William R. Bennett Prize Paper Award and the 1987 Outstanding IEEE Transactions of Automatic Control Paper Award. He has also received several institutional awards, including the Research Excellence Award in 1989, the Outstanding Achievement Award in 1999, the Distinguished Faculty Achievement Award in 2001, and the Stephen Attwood Award in 2004 from The University of Michigan (the highest honor bestowed to Michigan Engineering faculty); a Distinguished Alumni Award of the College of Engineering, Seoul National University in 2002; the 2003 IEEE RTC Technical Achievement Award; and the 2006 Ho-Am Prize in Engineering (the highest honor bestowed to Korean-origin engineers). $\mathrm{He}$ has chaired several major conferences, including 2009 ACM MobiCom, 2008 IEEE SECON, 2005 ACM/USENIX MobiSys, 2000 IEEE RTAS, and 1987 IEEE RTSS. He has served on editorial boards including the IEEE Transactions on Parallel and Distributed Systems and the ACM Transactions on Embedded Systems. He has also served or is serving on numerous government committees, such as the US National Science Foundation Cyber-Physical Systems Executive Committee and the Korean Government R\&D Strategy Advisory Committee. He has also cofounded a couple of startups. He is a fellow of both the IEEE and the ACM.

$\triangleright$ For more information on this or any other computing topic, please visit our Digital Library at www.computer.org/publications/dlib. 